\title{
Soliton solutions for space-time fractional Heisenberg ferromagnetic spin chain equation by generalized Kudryashov method and modified $\exp (-\Omega(\eta))$-expansion function method
}

\author{
S. Tuluce Demiray and U. Bayrakci \\ Department of Mathematics, Osmaniye Korkut Ata University, Osmaniye, Turkey. \\ *e-mail: seymatuluce@gmail.com; ubayrakci42@gmail.com
}

Received 12 November 2020; accepted 17 December 2020

\begin{abstract}
This paper addresses the Heisenberg ferromagnetic spin chain equation with beta derivative. Initially, beta derivatives and their features are presented. Then, by submitting the generalized Kudryashov method and modified $\exp (-\Omega(\eta))$-expansion function method, dark, bright, and dark-bright soliton solutions of this equation, which can be explained with beta derivative, are procured. Thus, it seems that these methods can supply significant outcomes in finding the exact solutions fractional differential equations with beta time derivative.
\end{abstract}

Keywords: Heisenberg ferromagnetic spin chain equation; generalized Kudryashov method; modified $\exp (-\Omega(\eta))$-expansion function method; dark soliton; bright soliton; dark-bright soliton; beta time derivative.

PACS: $35 \mathrm{C} 08-35 \mathrm{~N} 05-68 \mathrm{~N} 15$

DOI: https://doi.org/10.31349/RevMexFis.67.393

\section{Introduction}

In recent years, fractional differential equations (FDEs) shed light on the science environment because of their important position in many areas of complicated physical events, from fluid dynamics and optical fiber to quantum field theory. In fact, the soliton solutions of such equations have been one of the most remarkable solutions due to their more clear view of the nonlinear physical properties and then guide to the next aims. As a result, several methods have been used by many authors to calculate such solutions for better insight into the main properties of physical constructions in different media [1-5].

A lot of kinds of fractional derivative operators have been described by scientists. Some of them are Caputo derivative, Riemann-Liouville derivative, Caputo-Fabrizio, Jumarie's modified Riemann-Liouville derivative, AtanganaBaleanu derivative [6-9]. By using these derivative operators, many methods have been submitted to provide solutions of FDEs [10-15].

Then, the conformable derivative has been identified by Khalil et al. [16]. Also, exact solutions of the FDE have been found by using this derivative [17]. Then, some theorems, definitions, and properties related to conformable derivative have been presented by Atangana et al. [18]. Consequently, a new fractional derivative called beta-derivative has been given by Atangana et al. [19]. Then, solutions of FDEs with conformable derivatives have been considered by a lot of authors [20-25].

Heisenberg's ferromagnetic spin chain equation has been highly considered for its significance from different aspects [26-29]. This paper will address Heisenberg ferromagnetic spin chain equation to get its soliton solutions by a strong algorithm that was lately submitted. The methods are the gen- eralized Kudryashov method (GKM) [30-33] and modified $\exp (-\Omega(\eta))$-expansion function method (MEFM) [34-37]. The algorithms supply optical solitons such as dark, bright, and dark-bright. The results are thus found after the extensive experience of the algorithmic operation.

\section{Beta Derivatives and its features}

Definition 1. The conformable derivative has been identified by Khalil et al. [16]. Let $w:[0, \infty)$ be a function $\beta$-th order, the conformable derivative of $w(t)$ for all is given as follows:

$$
\begin{aligned}
F^{\beta}(w(t)) & =\frac{d^{\beta} w(t)}{d t^{\beta}}=\lim _{e \rightarrow 0} \frac{w\left(t+e t^{1-\beta}\right)-w(t)}{e}, \\
0 & <\beta \leq 1
\end{aligned}
$$

Also, if $w$ is $\beta$-differentiable in $(0, a), a>0$, and $\lim _{e \rightarrow 0^{+}} w^{(\beta)}(t)$ exists, then it can be written as $w^{(\beta)}(0)=$ $\lim _{e \rightarrow 0^{+}}(t)$.

Definition 2. Let $w(t)$ be a function qualified for all nonnegative $t$. Then, the beta derivative of $w(t)$ is defined by [19]

$$
\begin{aligned}
F^{\beta}(w(t)) & =\frac{d^{\beta} w(t)}{d t^{\beta}} \\
& =\lim _{e \rightarrow 0} \frac{w\left(t+e\left[t+\frac{1}{\Gamma(\beta)}\right]^{1-\beta}\right)-w(t)}{e}, \\
0 & <\beta \leq 1 .
\end{aligned}
$$

Although the conformable fractional derivative presented by Khalil et al. supplies some basic properties such as the 
chain rule, Atangana's fractional derivative is presented because it can yield the maximum features of the basic derivatives.

Such derivatives can not only be assumed as fractional derivatives but also considered as a natural extension of the classical derivative. There is a significant theorem for betaderivatives [19]:

Theorem. Let $w(t)$ and $v(t)$ be $\beta$-differentiable functions for all $t>0$ and $\beta \in(0,1]$. Then

$$
\begin{aligned}
F^{\beta}(m w(t)+n v(t)) & =m F^{\beta}(w(t))+n F^{\beta}(v(t)), \\
\forall m, n \in R . & \\
F^{\beta}(w(t) v(t)) & =v(t) F^{\beta}(w(t))+w(t) F^{\beta}(v(t)), \\
F^{\beta}\left(\frac{w(t)}{v(t)}\right) & =\frac{v(t) F^{\beta}(w(t))-w(t) F^{\beta}(v(t))}{(v(t))^{2}}, \\
F^{\beta}(w(t)) & =\left(t+\frac{1}{\Gamma(\beta)}\right)^{1-\beta} \frac{d w(t)}{d t} .
\end{aligned}
$$

\section{General structure of GKM}

We survey the following FDE with beta derivative for a function of two real variables, space $x$, and time $t$ :

$$
P\left(k, F^{\beta} k, k_{t}, k_{x}, k_{x x}, \cdots\right)=0
$$

Step 1. Initially, we should perform the traveling wave solution of Eq. (1) as follows;

$$
k(x, y, t)=H(\eta) e^{i \lambda(x, y, t)},
$$

where

$$
\begin{aligned}
\eta & =\frac{\cos \phi}{\beta}\left(x+\frac{1}{\Gamma(\beta)}\right)^{\beta}+\frac{\sin \phi}{\beta}\left(y+\frac{1}{\Gamma(\beta)}\right)^{\beta} \\
& +\frac{p}{\beta}\left(t+\frac{1}{\Gamma(\beta)}\right)^{\beta}, \\
\lambda(x, y, t) & =-\left(\frac{\cos \phi}{\beta}\left[x+\frac{1}{\Gamma(\beta)}\right]^{\beta}+\frac{\sin \phi}{\beta}\left[y+\frac{1}{\Gamma(\beta)}\right]^{\beta}\right) \\
& +\frac{\sigma}{\beta}\left(t+\frac{1}{\Gamma(\beta)}\right)^{\beta}
\end{aligned}
$$

where $p$ and $\sigma$ arbitrary constants. Then, by substituting Eqs. (2-4) to Eq. (1), a nonlinear ordinary differential equation can be obtained as:

$$
N\left(H, H^{\prime}, H^{\prime \prime}, H^{\prime \prime \prime}, \cdots\right)=0,
$$

where the prime displays differentiation about $\eta$.

Step 2. Assume that the exact solutions of Eq. (5) can be considered in the form

$$
k(\eta)=\frac{\sum_{i=0}^{N} a_{i} \gamma^{i}(\eta)}{\sum_{j=0}^{M} b_{j} \gamma^{j}(\eta)}=\frac{A(\gamma[\eta])}{B(\gamma[\eta])},
$$

where $\gamma(\eta)=1 / 1 \pm e^{\eta}$. We highlight that the function $\gamma$ is the solution of the equation:

$$
\gamma_{\eta}=\gamma^{\prime}=\gamma^{2}-\gamma
$$

Taking account of Eq. (6), we supply

$$
\begin{aligned}
k^{\prime}(\eta) & =\frac{A^{\prime} \gamma^{\prime} B-A B^{\prime} \gamma^{\prime}}{B^{2}}=\gamma^{\prime}\left(\frac{A^{\prime} B-A B^{\prime}}{B^{2}}\right) \\
& =\left(\gamma^{2}-\gamma\right)\left(\frac{A^{\prime} B-A B^{\prime}}{B^{2}}\right), \\
k^{\prime \prime}(\eta) & =\frac{\gamma^{2}-\gamma}{B^{2}}\left([2 \gamma-1]\left[A^{\prime} B-A B^{\prime}\right]+\frac{\gamma^{2}-\gamma}{B}\right. \\
& \left.\times\left[B\left\{A^{\prime \prime} B-A B^{\prime \prime}\right\}-2 B^{\prime} A^{\prime} B+2 A\left\{B^{\prime}\right\}^{2}\right]\right),
\end{aligned}
$$

Step 3. The solution of Eq. (5) can be defined as follows:

$$
k(\eta)=\frac{a_{0}+a_{1} \gamma+a_{2} \gamma^{2}+\cdots+a_{N} \gamma^{N}+\cdots}{b_{0}+b_{1} \gamma+b_{2} \gamma^{2}+\cdots+b_{M} \gamma^{M}+\cdots} .
$$

To compute the values $M$ and $N$ in Eq. (10) that is the pole order for the general solution of Eq. (5), we procure comparably as in the classical Kudryashov method on balancing the highest-order nonlinear terms in Eq. (5), and we can find a relation of $M$ and $N$. We can get values of $M$ and $N$.

Step 4. Substituting Eq. (6) into Eq. (5) ensures a polynomial $R(\gamma)$ of $\gamma$. Extracting the coefficients of $R(\gamma)$ to zero, we get a system of algebraic equations. Solving this system, we can identify $p$ and the variable coefficients of $a_{0}, a_{1}, a_{2}$, $\cdots a_{N}, b_{0}, b_{1}, b_{2}, \cdots, b_{M}$. Thus, we get the exact solutions to Eq. (5).

\section{Soliton solutions for Heisenberg ferromag- netic spin chain equation by GKM}

In this section, we look for exact solutions of the Heisenberg ferromagnetic spin chain equation with beta time derivative by using GKM.

It is helpful to use the spin to lie in a planet right angles to the chain axis. Thus, we consider Heisenberg ferromagnetic spin chain equation with beta time derivative [38]

$$
\begin{gathered}
i \frac{\partial^{\beta} k}{\partial t^{\beta}}-i \frac{\partial^{\beta} k}{\partial x^{\beta}}+\left(\frac{\partial^{2 \beta} k}{\partial x^{2 \beta}}+\frac{\partial^{2 \beta} k}{\partial y^{2 \beta}}\right) \\
-2 \frac{\partial^{2 \beta} k}{\partial x^{\beta} \partial y^{\beta}}+2|k|^{2} k=0 .
\end{gathered}
$$

Firstly, we take wave variable transformations as follows

$$
k(x, y, t)=H(\eta) e^{i \lambda(x, y, t)},
$$


where

$$
\begin{aligned}
\eta & =\frac{\cos \phi}{\beta}\left(x+\frac{1}{\Gamma(\beta)}\right)^{\beta}+\frac{\sin \phi}{\beta}\left(y+\frac{1}{\Gamma(\beta)}\right)^{\beta} \\
+ & \frac{p}{\beta}\left(t+\frac{1}{\Gamma(\beta)}\right)^{\beta}, \\
\lambda(x, y, t) & =-\left(\frac{\cos \phi}{\beta}\left[x+\frac{1}{\Gamma(\beta)}\right]^{\beta}+\frac{\sin \phi}{\beta}\left(y+\frac{1}{\Gamma(\beta)}\right)^{\beta}\right) \\
& +\frac{\sigma}{\beta}\left(t+\frac{1}{\Gamma(\beta)}\right)^{\beta} .
\end{aligned}
$$

Putting Eqs. (12-14) into Eq. (11) provides

$$
\begin{gathered}
(\cos \phi-\sin \phi)^{2} H^{\prime \prime}(\eta)-\left[(\cos \phi-\sin \phi)^{2}\right. \\
+\cos \phi+\sigma] H(\eta)+2 H^{3}(\eta)=0
\end{gathered}
$$

where $p=2 \sin 2 \phi-\cos \phi-2$.

For the balance principle between higher-order derivative $H^{\prime \prime}$ and highest power nonlinear terms $H^{3}$ in Eq. (15), one can be procured

$$
N-M+2=3 N-3 M \Rightarrow N=M+1 .
$$

By using GKM, the solution of Eq. (11) can be given as

$$
H(\eta)=\frac{a_{0}+a_{1} \gamma+a_{2} \gamma^{2}}{b_{0}+b_{1} \gamma}
$$

where $a_{0}, a_{1}$, and $a_{2}$ are found later and $\gamma(\eta)=\left(1 / 1 \pm e^{\eta}\right)$. The function $\gamma(\eta)$ provides as

$$
\gamma_{\eta}=\gamma^{\prime}=\gamma^{2}-\gamma
$$

Thus, the exact solutions of Eq. (11) are accessed as the following;

\section{Case 1.}

$$
\begin{aligned}
a_{0} & =\frac{1}{2} i(\cos \phi-\sin \phi) b_{0}, \\
a_{1} & =-\frac{a_{2}}{2}-i(\cos \phi-\sin \phi) b_{0} \\
b_{1} & =-\frac{a_{2}}{i(\cos \phi-\sin \phi)} \\
\sigma & =-\frac{1}{2} \cos \phi(2+3 \cos \phi) \\
& +3 \cos \phi \sin \phi-\frac{3 \sin ^{2} \phi}{2} .
\end{aligned}
$$

Replacing Eq. (19) into Eq. (17), dark soliton solutions of Eq. (11) can reached as

$$
\begin{aligned}
\kappa_{1}(x, y, t) & =\frac{1}{2} i(\cos \phi-\sin \phi) \tanh \left(\frac{1}{2}\left[\frac{\cos \phi}{\beta}\left\{x+\frac{1}{\Gamma(\beta)}\right\}^{\beta}+\frac{\sin \phi}{\beta}\left\{y+\frac{1}{\Gamma(\beta)}\right\}^{\beta}+\frac{p}{\beta}\left\{t+\frac{1}{\Gamma(\beta)}\right\}^{\beta}\right]\right) \\
& \times \exp \left(i \left[-\left\{\frac{\cos \phi}{\beta}\left(x+\frac{1}{\Gamma(\beta)}\right)^{\beta}+\frac{\sin \phi}{\beta}\left(y+\frac{1}{\Gamma(\beta)}\right)^{\beta}\right\}\right.\right. \\
& \left.\left.+\left\{-\frac{1}{2 \beta} \cos \phi(2+3 \cos \phi)+\frac{3}{\beta} \cos \phi \sin \phi-\frac{3 \sin ^{2} \phi}{2 \beta}\right\}\left\{t+\frac{1}{\Gamma(\beta)}\right\}^{\beta}\right]\right) \\
k_{2}(x, y, t) & =\frac{1}{2} i(\cos \phi-\sin \phi) \operatorname{coth}\left(\frac{1}{2}\left[\frac{\cos \phi}{\beta}\left\{x+\frac{1}{\Gamma(\beta)}\right\}^{\beta}+\frac{\sin \phi}{\beta}\left\{y+\frac{1}{\Gamma(\beta)}\right\}^{\beta}+\frac{p}{\beta}\left\{t+\frac{1}{\Gamma(\beta)}\right\}^{\beta}\right]\right) \\
& \times \exp \left(i \left[-\left\{\frac{\cos \phi}{\beta}\left(x+\frac{1}{\Gamma(\beta)}\right)^{\beta}+\frac{\sin \phi}{\beta}\left(y+\frac{1}{\Gamma(\beta)}\right){ }^{\beta}\right\}\right.\right. \\
& \left.\left.+\left\{-\frac{1}{2 \beta} \cos \phi(2+3 \cos \phi)+\frac{3}{\beta} \cos \phi \sin \phi-\frac{3 \sin ^{2} \phi}{2 \beta}\right\}\left\{t+\frac{1}{\Gamma(\beta)}\right\}^{\beta}\right]\right)
\end{aligned}
$$

\section{Case 2.}

$$
a_{0}=0, \quad a_{1}=-i(\cos \phi-\sin \phi) b_{1}, \quad a_{2}=i(\cos \phi-\sin \phi) b_{1}, \quad b_{0}=-\frac{b_{1}}{2}, \quad \sigma=-\cos \phi
$$


Replacing Eq. (22) into Eq. (17), bright soliton solutions of Eq. (11) can be determined as

$$
\begin{aligned}
\kappa_{3}(x, y, t) & =i(\cos \phi-\sin \phi) \operatorname{csch}\left(\frac{\cos \phi}{\beta}\left[x+\frac{1}{\Gamma(\beta)}\right]^{\beta}+\frac{\sin \phi}{\beta}\left[y+\frac{1}{\Gamma(\beta)}\right]^{\beta}+\frac{p}{\beta}\left[t+\frac{1}{\Gamma(\beta)}\right]^{\beta}\right) \\
& \times \exp \left(i\left[-\left\{\frac{\cos \phi}{\beta}\left(x+\frac{1}{\Gamma(\beta)}\right)^{\beta}+\frac{\sin \phi}{\beta}\left(y+\frac{1}{\Gamma(\beta)}\right)^{\beta}\right\}-\frac{\cos \phi}{\beta}\left\{t+\frac{1}{\Gamma(\beta)}\right\}^{\beta}\right]\right) \\
\kappa_{4}(x, y, t) & =i(\cos \phi-\sin \phi) \operatorname{sech}\left(\frac{\cos \phi}{\beta}\left[x+\frac{1}{\Gamma(\beta)}\right]^{\beta}+\frac{\sin \phi}{\beta}\left[y+\frac{1}{\Gamma(\beta)}\right]^{\beta}+\frac{p}{\beta}\left[t+\frac{1}{\Gamma(\beta)}\right]^{\beta}\right) \\
& \times \exp \left(i\left[-\left\{\frac{\cos \phi}{\beta}\left(x+\frac{1}{\Gamma(\beta)}\right)^{\beta}+\frac{\sin \phi}{\beta}\left(y+\frac{1}{\Gamma(\beta)}\right)\right\}-\frac{\cos \phi}{\beta}\left\{t+\frac{1}{\Gamma(\beta)}\right\}^{\beta}\right]\right)
\end{aligned}
$$

\section{Case 3.}

$$
\begin{aligned}
a_{0} & =\frac{1}{2} i(\cos \phi-\sin \phi) b_{1}, \quad a_{1}=-i(\cos \phi-\sin \phi) b_{1}, \quad a_{2}=i(\cos \phi-\sin \phi) b_{1}, \quad b_{0}=-\frac{b_{1}}{2}, \\
\sigma & =-\cos (\phi)(1+3 \cos (\phi))+6 \cos \phi \sin \phi-3 \sin ^{2} \phi
\end{aligned}
$$

Replacing Eq. (25) into Eq. (17), dark soliton solutions of Eq. (11) can be ascertained as

$$
\begin{aligned}
\kappa_{5}(x, y, t) & =-i(\cos \phi-\sin \phi) \operatorname{coth}\left(\frac{\cos \phi}{\beta}\left[x+\frac{1}{\Gamma(\beta)}\right]^{\beta}+\frac{\sin \phi}{\beta}\left[y+\frac{1}{\Gamma(\beta)}\right]^{\beta}+\frac{p}{\beta}\left[t+\frac{1}{\Gamma(\beta)}\right]^{\beta}\right) \\
& \times \exp \left(i \left[-\left\{\frac{\cos \phi}{\beta}\left(x+\frac{1}{\Gamma(\beta)}\right)^{\beta}+\frac{\sin \phi}{\beta}\left(y+\frac{1}{\Gamma(\beta)}\right)^{\beta}\right\}\right.\right. \\
& \left.\left.\left.+\left\{\frac{-\cos \phi(1+3 \cos \phi)+6 \cos \phi \sin \phi-3 \sin ^{2} \phi}{\beta}\right\} t+\frac{1}{\Gamma(\beta)}\right\}^{\beta}\right]\right) \\
\kappa_{6}(x, y, t) & =i(\cos \phi-\sin \phi) \tanh \left(\frac{\cos \phi}{\beta}\left[x+\frac{1}{\Gamma(\beta)}\right]^{\beta}+\frac{\sin \phi}{\beta}\left[y+\frac{1}{\Gamma(\beta)}\right]^{\beta}+\frac{p}{\beta}\left[t+\frac{1}{\Gamma(\beta)}\right]^{\beta}\right) \\
& \times \exp \left(i \left[-\left\{\frac{\cos \phi}{\beta}\left(x+\frac{1}{\Gamma(\beta)}\right)^{\beta}+\frac{\sin \phi}{\beta}\left(y+\frac{1}{\Gamma(\beta)}\right)^{\beta}\right\}\right.\right. \\
& \left.+\left\{\frac{-\cos \phi(1+3 \cos \phi)+6 \cos \phi \sin \phi-3 \sin ^{2} \phi}{\beta}\left\{t+\frac{1}{\Gamma(\beta)}\right\}^{\beta}\right]\right)
\end{aligned}
$$

\section{Case 4.}

$$
\begin{aligned}
a_{0} & =-\frac{1}{4} i(\cos \phi-\sin \phi) b_{1}, \quad a_{1}=i(\cos \phi-\sin \phi) b_{1}, \quad a_{2}=-i(\cos \phi-\sin \phi) b_{1}, \quad b_{0}=-\frac{b_{1}}{2}, \\
\sigma & =-\frac{1}{2} \cos \phi(2+3 \cos \phi)+3 \cos \phi \sin \phi-\frac{3 \sin ^{2} \phi}{2} .
\end{aligned}
$$


Putting Eq. (28) into Eq. (17), dark-bright soliton solutions of Eq. (11) can be provided as

$$
\begin{aligned}
\kappa_{7}(x, y, t) & =\frac{1}{2} i(\cos \phi-\sin \phi)\left(\left[-2+3 \operatorname{coth}\left\{\frac{\cos \phi}{\beta}\left(x+\frac{1}{\Gamma(\beta)}\right)^{\beta}+\frac{\sin \phi}{\beta}\left(y+\frac{1}{\Gamma(\beta)}\right)^{\beta}+\frac{p}{\beta}\left(t+\frac{1}{\Gamma(\beta)}\right)^{\beta}\right\}\right.\right. \\
& \left.+3 \operatorname{csch}\left\{\frac{\cos \phi}{\beta}\left(x+\frac{1}{\Gamma(\beta)}\right)^{\beta}+\frac{\sin \phi}{\beta}\left(y+\frac{1}{\Gamma(\beta)}\right)^{\beta}+\frac{p}{\beta}\left(t+\frac{1}{\Gamma(\beta)}\right)^{\beta}\right\}\right]-\frac{a_{1}}{b_{1}}\left[-1+\operatorname{coth}\left\{\frac { 1 } { 2 } \left(\frac{\cos \phi}{\beta}\right.\right.\right. \\
& \left.\left.\left.\left.\times\left\{x+\frac{1}{\Gamma(\beta)}\right]^{\beta}+\frac{\sin \phi}{\beta}\left\{y+\frac{1}{\Gamma(\beta)}\right]^{\beta}+\frac{p}{\beta}\left[t+\frac{1}{\Gamma(\beta)}\right]^{\beta}\right)\right\}\right]\right) \exp \left(i \left[-\left\{\frac{\cos \phi}{\beta}\left(x+\frac{1}{\Gamma(\beta)}\right)^{\beta}\right.\right.\right. \\
& \left.\left.\left.\left.+\frac{\sin \phi}{\beta}\left(y+\frac{1}{\Gamma(\beta)}\right)^{\beta}\right\}+\left\{-\frac{1}{2 \beta} \cos \phi(2+3 \cos \phi)+\frac{3}{\beta} \cos \phi-\frac{3 \sin ^{2} \phi}{2 \beta}\right\}\left\{t+\frac{1}{\Gamma(\beta)}\right\}\right\}^{\beta}\right]\right)
\end{aligned}
$$
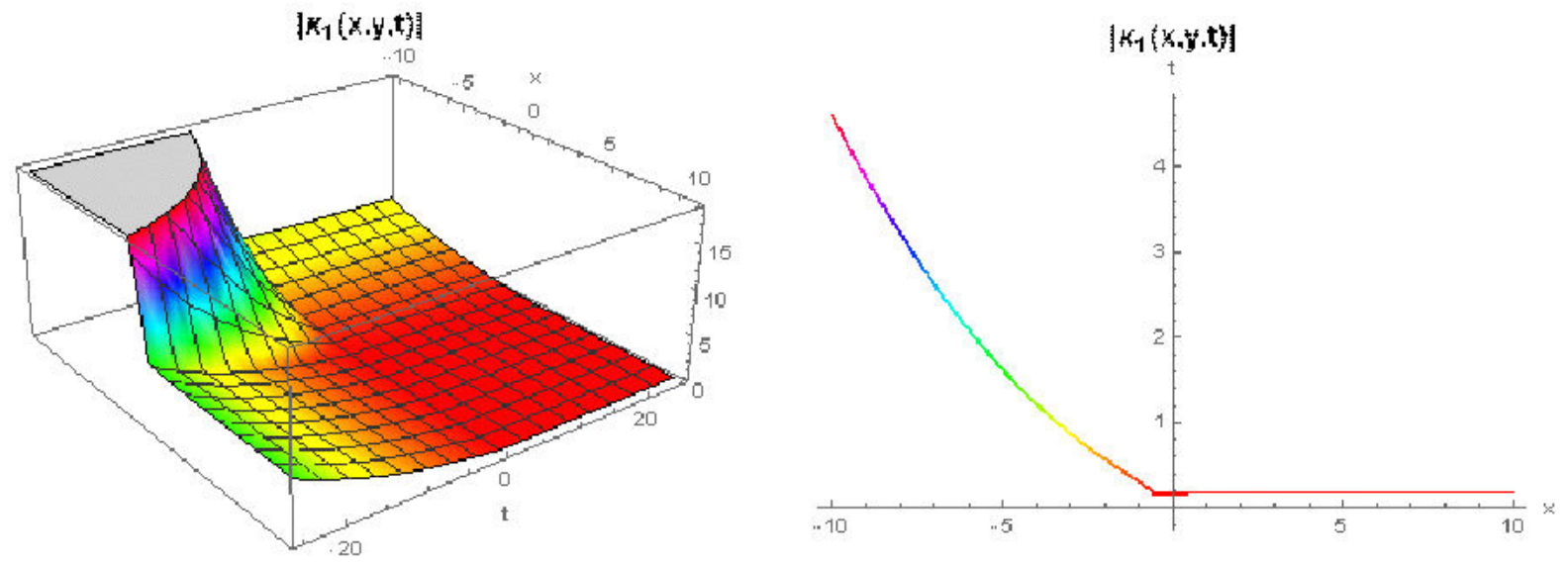

FIGURE 1. 3D image of $\left|\kappa_{1}(x, y, t)\right|$ for $\phi=60^{\circ}, \beta=0.5, y=1$ and 2D image of $\left|\kappa_{1}(x, y, t)\right|$ for these values and $t=0.2$.
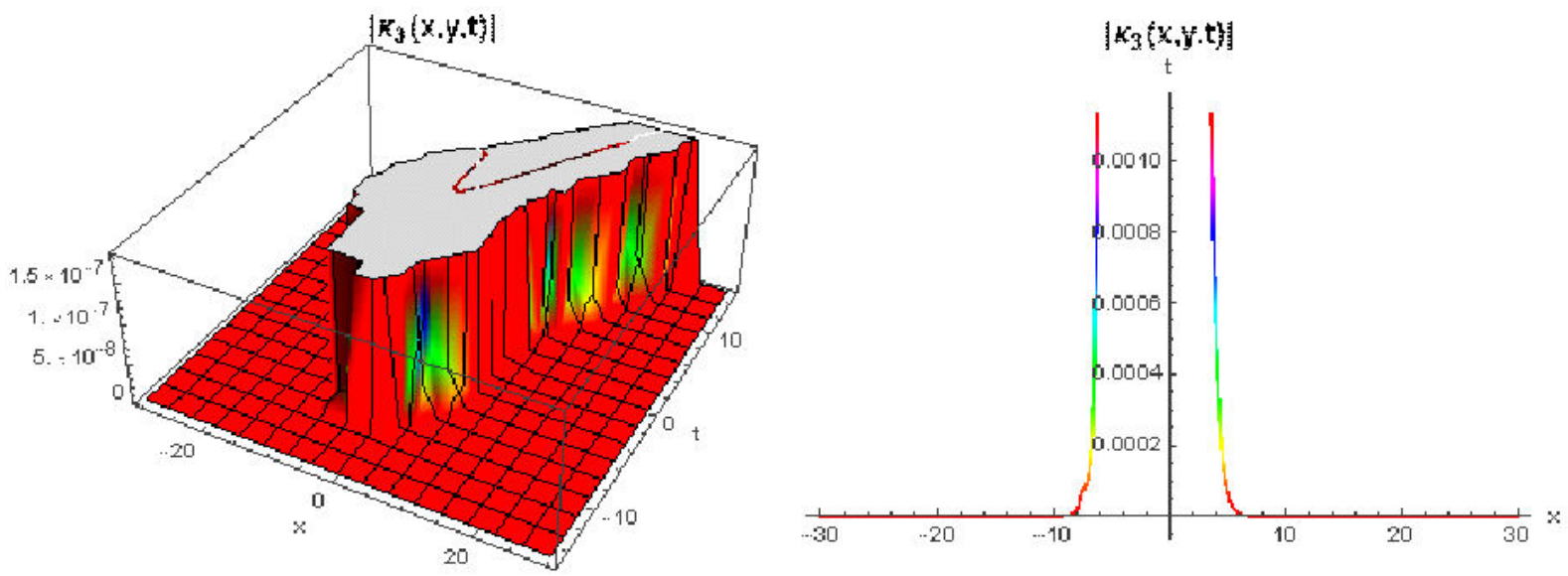

FIGURE 2. 3D image of $\left|\kappa_{3}(x, y, t)\right|$ for $\phi=30^{\circ}, \beta=1.5, y=2$ and 2D image of $\left|\kappa_{3}(x, y, t)\right|$ for these values and $t=0.4$. 


$$
\begin{aligned}
\kappa_{8}(x, y, t) & =\frac{1}{2} i(\cos \phi-\sin \phi)\left(\left[-2+3 \tanh \left\{\frac{\cos \phi}{\beta}\left(x+\frac{1}{\Gamma(\beta)}\right)^{\beta}+\frac{\sin \phi}{\beta}\left(y+\frac{1}{\Gamma(\beta)}\right)^{\beta}+\frac{p}{\beta}\left(t+\frac{1}{\Gamma(\beta)}\right)^{\beta}\right\}\right.\right. \\
& \left.+3 \operatorname{sech}\left\{\frac{\cos \phi}{\beta}\left(x+\frac{1}{\Gamma(\beta)}\right)^{\beta}+\frac{\sin \phi}{\beta}\left(y+\frac{1}{\Gamma(\beta)}\right)^{\beta}+\frac{p}{\beta}\left(t+\frac{1}{\Gamma(\beta)}\right)^{\beta}\right\}\right]-\frac{a_{1}}{b_{1}}\left[-1+\tanh \left\{\frac { 1 } { 2 } \left(\frac{\cos \phi}{\beta}\right.\right.\right. \\
& \left.\left.\left.\left.\times\left[x+\frac{1}{\Gamma(\beta)}\right]^{\beta}+\frac{\sin \phi}{\beta}\left\{y+\frac{1}{\Gamma(\beta)}\right]^{\beta}+\frac{p}{\beta}\left[t+\frac{1}{\Gamma(\beta)}\right]^{\beta}\right)\right\}\right]\right) \exp \left(i \left[-\left\{\frac{\cos \phi}{\beta}\left(x+\frac{1}{\Gamma(\beta)}\right)^{\beta}\right.\right.\right. \\
& \left.\left.\left.+\frac{\sin \phi}{\beta}\left(y+\frac{1}{\Gamma(\beta)}\right)^{\beta}\right\}+\left\{-\frac{1}{2 \beta} \cos \phi(2+3 \cos \phi)+\frac{3}{\beta} \cos \phi \sin \phi-\frac{3 \sin ^{2} \phi}{2 \beta}\right\}\left\{t+\frac{1}{\Gamma(\beta)}\right\}^{\beta}\right]\right)
\end{aligned}
$$

In Fig. 1, 3D and 2D graphs are investigated to illustrate the influence of the parameter $\beta$ on the dynamics of the first dark soliton solution. Clearly, the physical behavior of the dark soliton solution is altered when the parameter $\beta$ gets different values.

In Fig. 2, 3D and 2D graphs are examined to show the influence of the parameter $\beta$ on the dynamics of the bright soliton solution. Unquestionably, the physical behavior of the bright soliton solution is changed when the parameter $\beta$ takes different values.

\section{General structure of MEFM}

We survey the following FDE with beta derivative for a function of two real variables, space $x$, and time $t$ :

$$
P\left(\kappa, F^{\beta} \kappa, \kappa_{t}, \kappa_{x}, \kappa_{x x}, \cdots\right)=0 .
$$

Step 1. Initially, we should perform the traveling wave solution of Eq. (31) as follows;

$$
\kappa(x, y, t)=H(\eta) e^{i \lambda(x, y, t)},
$$

where

$$
\begin{aligned}
\eta & =\frac{\cos \phi}{\beta}\left(x+\frac{1}{\Gamma(\beta)}\right)^{\beta} \\
& +\frac{\sin \phi}{\beta}\left(y+\frac{1}{\Gamma(\beta)}\right)^{\beta}+\frac{p}{\beta}\left(t+\frac{1}{\Gamma(\beta)}\right)^{\beta}, \\
\lambda(x, y, t) & =-\left(\frac{\cos \phi}{\beta}\left[x+\frac{1}{\Gamma(\beta)}\right]^{\beta}\right. \\
+ & \left.\frac{\sin \phi}{\beta}\left[y+\frac{1}{\Gamma(\beta)}\right]^{\beta}+\frac{\sigma}{\beta}\left[t+\frac{1}{\Gamma(\beta)}\right]^{\beta}\right),
\end{aligned}
$$

where $p$ and $\sigma$ arbitrary constants. Then, by substituting Eqs. (32-34) to Eq. (31), a nonlinear ordinary differential equation can be obtained as:

$$
N\left(H, H^{\prime}, H^{\prime \prime}, H^{\prime \prime \prime}, \cdots\right)=0,
$$

where the prime displays differentiation about $\eta$.
Step 2: Presume the traveling wave solution of Eq. (4) can be indicated as follows:

$$
\begin{aligned}
& \kappa(\eta)=\frac{\sum_{i=0}^{N} F_{i}(\exp [-\Omega\{\eta\}])^{i}}{\sum_{j=0}^{M} G_{j}(\exp [-\Omega\{\eta\}])^{j}} \\
& =\frac{F_{0}+F_{1} \exp (-\Omega)+\cdots+F_{N} \exp (N(-\Omega))}{G_{0}+G_{1} \exp (-\Omega)+\cdots+G_{M} \exp (M(-\Omega))},
\end{aligned}
$$

where $F_{i}, G_{j},(0 \leq i \leq N, 0 \leq j \leq M)$ are constants to be described later, such that $F_{N} \neq 0, G_{M} \neq 0$, and $\Omega=\Omega(\eta)$ is the solution of the following ordinary differential equation:

$$
\Omega^{\prime}(\eta)=\exp (-\Omega[\eta])+n \exp (\Omega[\eta])+m .
$$

The solution families of Eq. (37) can be shown as follows: Family1: If $n \neq 0, m^{2}-4 n>0$,

$$
\begin{aligned}
\Omega(\eta) & =\ln \left(\frac{-\sqrt{m^{2}-4 n}}{2 n} \tanh \right) \\
& \left.\times\left[\frac{\sqrt{m^{2}-4 n}}{2 n}\{\eta+E\}\right]-\frac{m}{2 n}\right) .
\end{aligned}
$$

Family2: If $n \neq 0, m^{2}-4 n<0$,

$$
\begin{aligned}
\Omega(\eta) & =\ln \left(\frac{\sqrt{-m^{2}+4 n}}{2 n} \tan \right) \\
& \left.\times\left[\frac{\sqrt{-m^{2}+4 n}}{2}\{\eta+E\}\right]-\frac{m}{2 n}\right) .
\end{aligned}
$$

Family3: If $n=0, m \neq 0$, and $m^{2}-4 n>0$,

$$
\Omega(\eta)=-\ln \left(\frac{m}{\exp [m\{\eta+E\}]-1}\right) .
$$

Family4: If $n \neq 0, m \neq 0$, and $m^{2}-4 n=0$,

$$
\Omega(\eta)=\ln \left(-\frac{2 m[\eta+E]+4}{m^{2}[\eta+E]}\right) .
$$


Family5: If $n=0, m=0$, and $m^{2}-4 n=0$,

$$
\Omega(\eta)=\ln (\eta+E) .
$$

The positive integers $M$ and $M$ can be determined attending the homogeneous balance principle in Eq. (36).

Step 3: Replacing Eqs. (37) and (38-42) into Eq. (36), we ascertain a polynomial of $\exp (-\Omega(\eta))$. We stabilize all the coefficients of same power of $\exp (-\Omega(\eta))$ to zero. This operation determines a system of equations that can be unfastened to reach $F_{0}, F_{1}, F_{2}, \cdots F_{N}, G_{0}, G_{1}, G_{2}, \cdots G_{M}$, $E, m, n$ by the way of Wolfram Mathematica 12 . Inserting the values of these constants into Eq. (36), the general solutions of Eq. (36) supplies the determination of the solution of Eq. (31).

\section{Soliton solutions for Heisenberg ferromag- netic spin chain equation by MEFM}

In this section, we seek exact solutions of the Heisenberg ferromagnetic spin chain equation with beta time derivative by using MEFM.

For the balance principle between higher-order derivative $H^{\prime \prime}$ and highest power nonlinear terms $H^{3}$ in Eq. (15), one can procure

$$
N=M+1 \text {. }
$$

By using MEFM, the solution of Eq. (11) can be given as

$$
H=\frac{F_{0}+F_{1} \exp (-\Omega)+F_{2} \exp (2[-\Omega])+}{G_{0}+G_{1} \exp (-\Omega)}=\frac{Z}{\tau},
$$

and

$$
\begin{aligned}
H^{\prime} & =\frac{Z^{\prime} \tau-\tau^{\prime} Z}{\tau^{2}} \\
H^{\prime \prime} & =\frac{Z^{\prime \prime} \tau^{3}-\tau^{2} Z^{\prime} \tau^{\prime}-\left(\tau^{\prime \prime} Z+\tau^{\prime} Z^{\prime}\right) \tau^{2}+2\left(\tau^{\prime}\right)^{2} Z \tau}{\tau^{4}}
\end{aligned}
$$

where $F_{2} \neq 0$ and $G_{1} \neq 0$. The function $\Omega=\Omega(\eta)$ provides as

$$
\Omega^{\prime}(\eta)=\exp (-\Omega[\eta])+n \exp (\Omega[\eta])+m
$$

Thus, the exact solutions of Eq. (11) are accessed as the following;

\section{Case 1:}

$$
\begin{aligned}
F_{0}= & \frac{1}{2} i(\cos \phi-\sin \phi) m G_{0}, \\
F_{1}= & \frac{1}{2} i(\cos \phi-\sin \phi)\left(2 G_{0}+m G_{1}\right), \\
F_{2}= & i(\cos \phi-\sin \phi) G_{1}, \\
\sigma & =\cos \phi\left(-1+\sin \phi\left[2+m^{2}-4 n\right]\right) \\
& -\frac{1}{2}\left(2+m^{2}-4 n\right) .
\end{aligned}
$$

Replacing Eq. (48) into Eq. (44), dark soliton solutions of Eq. (11) can be reached as

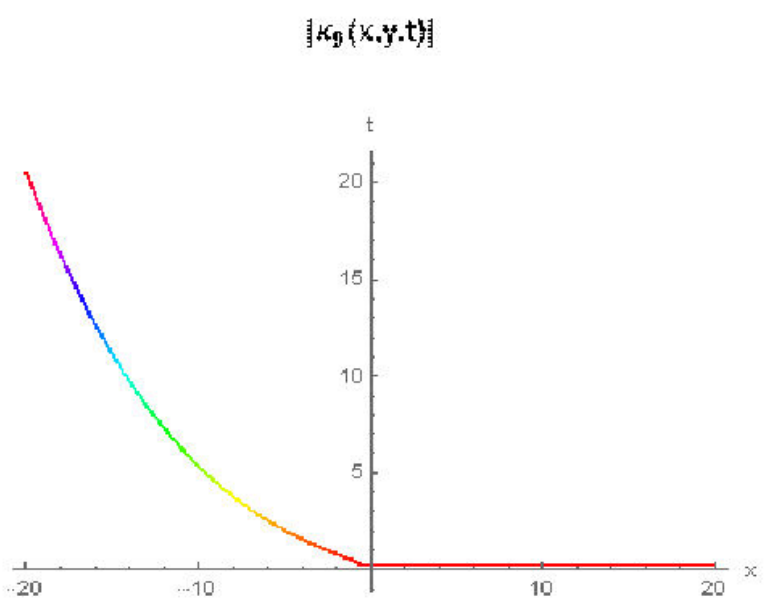

FIGURE 3. 3D image of $\left|\kappa_{9}(x, y, t)\right|$ for $\phi=60^{\circ}, \beta=0.5, y=3, m=0.5, n=-0.4, E=0.3$, and $2 \mathrm{D}$ image of $\left|\kappa_{9}(x, y, t)\right|$ for these values and $t=0.7$. 


$$
\begin{aligned}
& =\frac{1}{2} i(\cos \phi-\sin \phi) \frac{J+m \sqrt{J} \tanh \left(\frac{\sqrt{J}\left[\cos \phi\left\{x+\frac{1}{\Gamma(\beta)}\right\}^{\beta}+\sin \phi\left\{y+\frac{1}{\Gamma(\beta)}\right\}^{\beta}+p\left\{t+\frac{1}{\Gamma(\beta)}\right\}^{\beta}\right]+E \beta}{2 \beta}\right)}{m+\sqrt{J} \tanh \left(\frac{\sqrt{J}\left[\cos \phi\left\{x+\frac{1}{\Gamma(\beta)}\right\}^{\beta}+\sin \phi\left\{y+\frac{1}{\Gamma(\beta)}\right\}^{\beta}+p\left\{t+\frac{1}{\Gamma(\beta)}\right\}^{\beta}\right]+E \beta}{2 \beta}\right)} \\
& \times \exp \left(i \left[-\left\{\frac{\cos \phi}{\beta}\left(x+\frac{1}{\Gamma(\beta)}\right)^{\beta}+\frac{\sin \phi}{\beta}\left(y+\frac{1}{\Gamma(\beta)}\right)^{\beta}\right\}\right.\right. \\
& \left.\left.+\left\{\frac{\cos \phi}{\beta}\left(-1+\sin \phi\left[2+m^{2}-4 n\right]-\frac{1}{2 \beta}\left[2+m^{2}-4 n\right]\right)\right\}\left\{t+\frac{1}{\Gamma(\beta)}\right\}^{\beta}\right]\right)
\end{aligned}
$$

where $n \neq 0$, and $J=m^{2}-4 n>0$.

In Fig. 3, 3D and 2D graphs are considered to indicate the influence of the parameter on the dynamics of the dark soliton solution. Explicitly, the physical behavior of the dark soliton solution is shifted when the parameter $\beta$ receives different values.

Case 2:

$$
\begin{aligned}
G_{0} & =\frac{r-s}{\sqrt{2} \sqrt{F_{1}^{2}-2 F_{0} F_{2}-F_{1} \sqrt{F_{1}^{2}-4 F_{0} F_{2}}}}, \\
G_{1} & =\frac{F_{1}-\sqrt{F_{1}^{2}-4 F_{0} F_{2}}}{2 \sqrt{2} F_{0}} \frac{\sqrt{F_{1}^{2}-2 F_{0} F_{2}-F_{1} \sqrt{F_{1}^{2}-4 F_{0} F_{2}}}}{r-s} \\
R & =\frac{F_{1}-\sqrt{F_{1}^{2}-4 F_{0} F_{2}}}{F_{2}}, \quad r=\cos \phi, \quad s=\sin \phi, \\
\sigma & =\frac{-(r-s)^{2} F_{1}^{2}+2(r-s)^{2} F_{0} F_{2}+\left(-r-r^{2}+2 r s-s^{2}+2(r-s)^{2} S\right) F_{2}^{2}+(r-s)^{2} F_{1} \sqrt{F_{1}^{2}-4 F_{0} F_{2}}}{F_{2}^{2}} .
\end{aligned}
$$

Replacing Eq. (50) into Eq. (44), dark soliton solutions of Eq. (11) can be reached as

$\kappa_{10}(x, y, t)=$

$$
=\frac{A\left(F_{2}+\frac{1}{2 n}\left[-\frac{F_{1}-\sqrt{F_{1}^{2}-4 F_{0} F_{2}}}{F_{2}}-\chi \tanh \{f(x, y, t)\}\right]\left[F_{1}+\frac{F_{0}\left\{-\frac{F_{1}-\sqrt{F_{1}^{2}-4 F_{0} F_{2}}}{F_{2}}-\chi \tanh [f\{x, y, t\}]\right\}}{2 n}\right]\right.}{B(C-D \tanh [f\{x, y, t\}])\left(\frac{F_{1}-\sqrt{F_{1}^{2}-4 F_{0} F_{2}}}{F_{2}}+\chi \tanh [f\{x, y, t\}]\right)}
$$

where,

$$
\begin{aligned}
A & =4 i \sqrt{2}(r-s)^{2} n^{2} F_{0} F_{1} F_{2}, \quad B=\sqrt{\frac{F_{1} \sqrt{F_{1}^{2}-4 F_{0} F_{2}}+\left(F_{1}^{2}-2 F_{0} F_{2}\right)}{(r-s)^{2}},} \\
C & =-(r-s)^{2} F_{1}^{2}\left(F_{0}-n F_{2}\right)+\left(F_{0}-S F_{2}\right)(r-s)^{2} F_{1} \sqrt{F_{1}^{2}-4 F_{0} F_{2}} \\
D & =-\sqrt{2}(r-s)^{2} F_{0} F_{1} F_{2} \sqrt{\frac{F_{1}^{2}-2 F_{2}\left(F_{0}+n F_{2}\right)-F_{1} \sqrt{F_{1}^{2}-4 F_{0} F_{2}}}{F_{2}^{2}}} \\
f(x, y, t) & =\frac{\chi\left(\cos \phi\left[x+\frac{1}{\Gamma(\beta)}\right]^{\beta}+\sin \phi\left[y+\frac{1}{\Gamma(\beta)}\right]^{\beta}+p\left[t+\frac{1}{\Gamma(\beta)}\right]^{\beta}\right)+E \beta}{2 \beta} \\
\chi & =\sqrt{\frac{-4 n+\left(F_{1}^{2}-F_{1} \sqrt{F_{1}^{2}-4 F_{0} F_{2}}\right)^{2}}{F_{1}^{2} F_{2}^{2}}}
\end{aligned}
$$


and

$$
\frac{-4 n+\left(F_{1}^{2}-F_{1} \sqrt{F_{1}^{2}-4 F_{0} F_{2}}\right)^{2}}{F_{1}^{2} F_{2}^{2}}>0 .
$$

\section{Conclusion}

In this work, the soliton characters of the Heisenberg ferromagnetic spin chain equation with beta time derivative were investigated by using GKM and MEFM. Dark, bright, and dark-bright soliton solutions of this equation have been accomplished found. Then, 3D and 2D images were presented for some solutions, which display the vitality of the solutions with proper values. Numerical results, together with the graphical demonstrations, have exhibited the reliability of these methods. Also, these solutions have been reported to the literature with novel substantial physical properties. These methods can be applied to other FDEs with beta time derivatives.

1. B. Cuahutenango-Barro, M. A. Taneco-Hernandez and J. F. Gomez-Aguilar, On the solutions of fractional time wave equation with memory effect involving operators with regular kernel, Chaos. Solitons Fractals. 115 (2018) 283, https:// doi.org/10.1016/j.chaos.2018.09.002

2. I. Podlubny, Fractional Differential equations: an introduction to fractional derivatives, fractional differential equations, to methods of their solution and some of their applications, New York(NY), Academic Press, 1998, p. 198.

3. K. B. Oldham, J. Spanier, The fractional calculus, New York (NY), Academic Press, 1974.

4. J. Singh, D. Kumar, M. Al Qurashi and D. Baleanu, A new fractional model for giving up smoking Dynamics, $A d v$. Differ. Equ. 88 (2017) 1, https://doi.org/10.1186/ s13662-017-1139-9

5. M. Caputo and F.Mainardi, A new dissipation model based on memory mechanism, Pure Appl. Geophys. 91 (1971) 134, https://doi.org/10.1007/BF00879562.

6. R. Metzler and J. Klafter, The random walk's guide to anomalous diffusion: a fractional dynamics approach, Phys. Rep. 339 (2000) 1, https://doi.org/10.1016/ S0370-1573(00)00070-3.

7. M. Caputo and M. Fabrizio, A new definition of fractional derivative without singular kernel. Progr. Fract. Differ.Appl., 1 (2015) 73, http://doi.org/10.12785/ pfda/010201

8. G. Jumarie, Modified Riemann-Liouville derivative and fractional Taylor series of nondifferentiable functions further results, Comput. Math. Appl., 51 (2006) 1367, https://doi. org/10.1016/j.camwa.2006.02.001

9. A. Atangana and D. Baleanu, New fractional derivatives with nonlocal and non-singular kernel: theory and application to heat transfer model. Therm. Sci., 20 (2016) 763, https: //doi.org/10.2298/TSCI160111018A

10. S. Zhang and H.Q. Zhang, Fractional sub-equation method and its applications to nonlinear fractional PDEs. Phys. Lett. A, 375 (2011) 1069, https://doi.org/10.1016/j. physleta.2011.01.029

11. B. Lu, The first integral method for some time fractional differential equations. J. Math. Anal. Appl., 395 (2012) 684, https : //doi.org/10.1016/j.jmaa.2012.05.066
12. N. Das, R. Singh, A.M. Wazwaz and J. Kumar, An algorithm based on the variational iteration technique for the Bratu-type and the Lane-Emden problems. J. Math. Chem., 54 (2016) 527, https://doi.org/10.1007/s10910-015-0575-6

13. X. J. Yang and Y.D. Zhang, A new Adomian decomposition procedure scheme for solving local fractional Volterra integral equation. Adv. Inf. Tech. Manag., 1 (2012) 158.

14. H. Jafari and H.K. Jassim, Numerical solutions of telegraph and Laplace equations on cantor sets using local fractional Laplace decomposition method. Int. J. Adv. Appl. Math. Mech., 2 (2015) 144.

15. M.S. Hu, R.P. Agarwal and X.J. Yang, Local fractional Fourier series with application to wave equation in fractal vibrating string. Abstract. Appl. Anal., 2012 (2012) 567401, https : //doi.org/10.1155/2012/567401

16. R. Khalil, M. Al Horani, A. Yousef and M. Sababheh, A new definition of fractional derivative. J. Comput. Appl. Math., 264 (2014) 65, https://doi.org/10.1016/j.cam. 2014.01 .002

17. Y. Cenesiz and A. Kurt, The solution of time fractional heat equation with new fractional derivative definition. Recent Advances in Applied Mathematics, Modelling and Simulation, ISBN: 978-960-474-398-8, 195.

18. A. Atangana, D. Baleanu and A. Alsaedi, New properties of conformable derivative. Open Math., 13 (2015) 889, https : //doi.org/10.1515/math-2015-0081.

19. A. Atangana, D. Baleanu and A. Alsaedi, Analysis of time fractional Hunter-Saxton equation: a model of neumatic liquid crystal. Open Phys., 14 (2016) 145, https://doi.org/ $10.1515 /$ phys $-2016-0010$

20. W.S. Chung, Fractional Newton mechanics with conformable fractional derivative. J. Comput. Appl. Math., 290 (2015) 150, https://doi.org/10.1016/j.cam.2015.04.049

21. H. Yepez-Martinez, J.F. Gomez-Aguilar and A. Atangana, First integral method for non-linear differential equations with conformable derivative. Math. Model. Nat. Phenom., 13 (2018) 1, https://doi.org/10.1051/mmnp/2018012 
22. H. Yepez-Martinez and J.F. Gomez-Aguilar, Fractional subequation method for Hirota-Satsuma-coupled KdV equation and coupled mKdV equation using the Atangana's conformable derivative. Waves Random Complex Media, 29 (2019) 678, https://doi.org/10.1080/17455030.2018. 1464233

23. H. Yepez-Martinez and J.F. Gomez-Aguilar, Optical solitons solution of resonance nonlinear Schrödinger type equation with Atangana's-conformable derivative using sub-equation method. Waves Random Complex Media, (2019) 1, https://doi. org/10.1080/17455030.2019.1603413

24. S. Tuluce Demiray, New Soliton Solutions of Optical Pulse Envelope E(Z, $\tau)$ with Beta Time Derivative, Optik, 223 (2020) 1, https://doi.org/10.1016/j.ijleo. 2020.165453

25. S. Tuluce Demiray, New solutions of Biswas-Arshed equation with beta time derivative, Optik, 222 (2020) 1, https: //doi.org/10.1016/j.ijleo.2020.165405

26. M. M. Latha and C. C. Vasanthi, An integrable model of (2+1)dimensional Heisenberg ferromagnetic spin chain and soliton excitations, Phys. Scr. 89 (2014) 1, https://doi.org/ $10.1088 / 0031-8949 / 89 / 6 / 065204$

27. X. H. Zhao, B. Tian, D. Y. Liu, X. Y. Wu, J. Chai and Y. J. Guo, Dark solitons interaction for a $(2+1)$-dimensional nonlinear Schrödinger equation in the Heisenberg ferromagnetic spin chain, Superlattice Microst. 100 (2016) 587, https: //doi.org/10.1016/j.spmi.2016.10.014

28. B. Q. Li and Y. L. Ma, Lax pair, Darboux transformation and Nth-order rogue wave solutions for a $(2+1)$-dimensional Heisenberg ferromagnetic spin chain equation, Comput Math Appl. 77 (2019) 514, https://doi.org/10.1016/j. camwa.2018.09.054

29. M. S. Hashemi, Some new exact solutions of $(2+1)-$ dimensional nonlinear Heisenberg ferromagnetic spin chain with the conformable time fractional derivative, Opt Quant Electron. 50 (2018) 1, https://doi.org/10.1007/ s11082-018-1343-1.
30. S. Tuluce Demiray and H. Bulut, Soliton solutions of some nonlinear evolution problems by GKM, Neural Comput \& Applic, 31 (2019) 287, https://doi.org/10.1007/ s00521-017-2999-3

31. S. Tuluce Demiray, Y.Pandir and H. Bulut, New Soliton Solutions for Sasa-Satsuma Equation, Waves Random Complex Media. 25 (2015) 417, https://doi.org/10.1080/ 17455030.2015 .1042945 .

32. S. Tuluce Demiray, Y. Pandir and H. Bulut, New Solitary Wave Solutions of Maccari System, Ocean Eng. 103 (2015) 153, https://doi.org/10.1016/j.oceaneng.2015. 04.037 .

33. S. Tuluce Demiray, Y. Pandir and H. Bulut, All Exact Travelling Wave Solutions of Hirota Equation and Hirota-Maccari System, Optik, 127 (2016) 1848, https ://doi.org/10. $1016 / j . i j l e 0.2015 .10 .235$.

34. S. Tuluce Demiray and H. Bulut, Analytical Solutions of Phi-four equations, Int. J. Optim. Control, Theor. Appl. 7 (2017) 275, https://doi.org/10.11121/ijocta. 01.2017 .00493 .

35. S. Tuluce Demiray and H. Bulut, A New Method for (4+1) Dimensional Fokas Equation, ITM Web of Conf. 22 (2018) 1, https://doi.org/10.1051/itmconf/ 20182201065.

36. S. Tuluce Demiray and H. Bulut, Hyperbolic Function Solutions for Positive Gardner-KP Equation, J. Adv. Math. Stud. 12 (2019) 101.

37. S. Tuluce Demiray, H. Bulut, A New Approach for NizhnikNovikov-Veselov System, Advanced Mathematical Models \& Applications, 4 (2019) 79.

38. M. F. Uddin, M. G. Hafez, Z. Hammouch and D. Baleanu, Periodic and rogue waves for Heisenberg models of ferromagnetic spin chains with fractional beta derivative evolution and obliqueness, Waves Random Complex Media, (2020) 1, https://doi.org/10.1080/17455030. 2020.1722331. 